\title{
Anti-CHH Antibody Causes Impaired Hyperglycemia in Penaeus monodon
}

\author{
Supattra Treerattrakool ${ }^{1}$, Apinunt Udomkit ${ }^{1, *}$ and Sakol Panyim ${ }^{1,2}$ \\ ${ }^{1}$ Institute of Molecular Biology and Genetics, Mahidol University, Salaya Campus, Nakhon Pathom 73170, Thailand \\ ${ }^{2}$ Department of Biochemistry, Faculty of Science, Mahidol University, Bangkok 10400, Thailand
}

Received 31 August 2005, Accepted 20 March 2006

\begin{abstract}
Crustacean hyperglycemic hormone (CHH) plays a major role in controlling glucose level in the haemolymph and also triggers important events during molting and reproductive cycles. In Penaeus monodon, three types of $\mathrm{CHH}$, namely Pem-CHH1, Pem-CHH2 and Pem-CHH3, have been previously characterized. In this study, mouse polyclonal antibody was raised against recombinant Pem-CHH1 that was expressed in Escherichia coli. The anti-Pem-CHH1 antibody recognized all three types of Pem-CHHs but did not cross-react with either related hormone, moltinhibiting hormone of $\boldsymbol{P}$. monodon, or unrelated human growth hormone. The hyperglycemic activity in the extract from the eyestalk neural tissues was significantly depleted after incubating with anti-Pem-CHH antibody. Direct injection of the antibody into shrimp caused about $30-50 \%$ reduction in the haemolymph glucose level. The result demonstrates the ability of anti-Pem-CHH1 antibody to deplete the activity of $\mathrm{CHH}$ in vivo, and thus provides a possibility of using anti-Pem-CHH1 antibody to inhibit the hormone activity as a strategy to modulate growth and reproduction in this species.
\end{abstract}

Keywords: Antibody, Black tiger shrimp, Crustacean hyperglycemic hormone, Eyestalk, Sinus gland

\section{Introduction}

Crustacean hyperglycemic hormone $(\mathrm{CHH})$ is the most abundant hormone produced by the X-organ in crustacean eyestalk. It plays a major role in controlling sugar level in the haemolymph (Cooke and Sullivan, 1982). CHH is a member of a peptide family, socalled the $\mathrm{CHH}$ family that is composed of $\mathrm{CHH}$ and the other three hormones: molt-inhibiting hormone (MIH); gonadinhibiting hormone (GIH); mandibular organ-inhibiting hormone

\footnotetext{
* To whom correspondence should be addressed.

Tel: 66-2-800-3624 ext 1236; Fax: 66-2-441-9906

E-mail: staud@mahidol.ac.th
}

(MO-IH) (Charmantier et al., 1997). The CHH-family peptides harbor special features among themselves. They are composed of 72-80 amino acid residues with six cysteine residues aligned at conserved positions (Keller, 1992). The hormones of similar characteristics to the $\mathrm{CHH}$ family have also been identified in species other than crustacean. For example, the $\mathrm{CHH}$-like peptide of the silk worm, Bombyx mori (Endo et al., 2000) and the ion transport peptide (ITP) of the locust, Schistocerca gregaria (Meredith et al., 1995).

The hyperglycemic activity of $\mathrm{CHH}$ through the elevation of glucose level in the haemolymph is presumably exerted by stimulation of glycogen breakdown, the reaction mediated by glycogen phosphorylase, and by inhibitory effect on glycogen synthase (Sedlmeier, 1982; Chang and O'Connor, 1985). In addition, the level of cAMP and cGMP in the abdominal muscle of Orconectes limosus was elevated after injection with $\mathrm{CHH}$ (Sedlmeier, 1982) suggesting similarity between the mode of action of $\mathrm{CHH}$ and those of vertebrate peptide hormones that occur via cAMP or cGMP as a second messenger.

A number of evidences have demonstrated other physiological roles of $\mathrm{CHH}$ apart from its principle role in glucose metabolism. For instance, $\mathrm{CHH}$ was involved in osmoregulation in the American lobster, Homarus americanus (CharmantierDaures et al., 1994) and the crayfish, Astacus leptodactylus (Serrano et al., 2003). Lipid metabolism in several crustaceans was also shown to be affected by CHH (Santos et al., 1997). Additionally, it has been clearly demonstrated that $\mathrm{CHH}$ was involved in the mediation of ecdysteroid synthesis (Yasuda et al., 1994) as well as ovarian protein synthesis (Khayat et al., 1998; Avarre et al., 2001).

The recombinant peptide hormones with hyperglycemic activity have been expressed from the cDNA isolated from the optic ganglia of the black tiger shrimp, Penaeus monodon (Treerattrakool et al., 2003; Udomkit et al., 2004). The aim of our research here is the production of specific antibody against Pem-CHH1 of $P$. monodon for studying whether its binding to $\mathrm{CHH}$ can inhibit the function of the hormone. Furthermore, the anti-CHH antibody was used for injection into $P$. monodon in order to examine its effect on the activity of the hormone in the shrimp. 


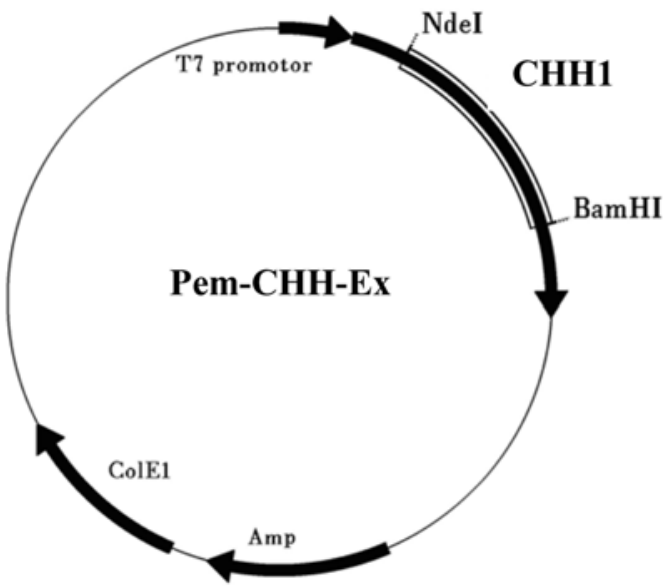

Fig. 1. Physical map of Pem-CHH-Ex expression plasmid. A cDNA encoding the mature peptide region of Pem-CHH1 was cloned into pET-3a vector at $\mathrm{NdeI}$ and BamHI sites downstream of the $\mathrm{T} 7$ promoter.

\section{Materials and Methods}

Expression of recombinant Pem-CHH1 in Escherichia coli. A single colony of $E$. coli transformant containing Pem-CHH-EX in which the cDNA encoding the mature Pem-CHH1 had been inserted into pET3a vector (Fig. 1) was grown overnight at $37^{\circ} \mathrm{C}$ in LB medium supplemented with $100 \mu \mathrm{g} / \mathrm{ml}$ ampicillin and $34 \mu \mathrm{g} / \mathrm{ml}$ chloramphenical. The overnight culture was diluted $(1: 100)$ with the same medium and incubated at $37^{\circ} \mathrm{C}$ with vigorous shaking to an $\mathrm{OD}_{600}$ of $0.5-0.7$. Isopropyl-1-thio- $\beta$-D-galactoside (IPTG) was added to the culture to a final concentration of $0.4 \mathrm{mM}$, and the incubation was continued for $4 \mathrm{~h}$. Then the bacterial cells were harvested and resuspended in $1 / 20$ culture volume of cold distilled water. After breaking the cells in a French ${ }^{\circledR}$ Pressure Cell Press $\left(\mathrm{AMINGO}^{\circledR}, \mathrm{USA}\right)$ at 900 psi, the supernatant was removed and the pellet was washed with cold distilled water for 5 times.

Purification of recombinant Pem-CHH1. The inclusion bodies of Pem-CHH1 were solubilized in $8 \mathrm{M}$ Urea in PBS, $\mathrm{pH}$ 7.4. The resulting solubilized fraction was loaded into a Superdex-200 (HR10/30, Global) gel filtration column that was connected to ÄKTA Purification system (Amersham Phamacia Biotech, USA). The column was eluted with 2 column volumes of $8 \mathrm{M}$ Urea in PBS, $\mathrm{pH}$ 7.4 at the flow rate of $0.25 \mathrm{ml} / \mathrm{min}$. Finally, The elution buffer was replaced by extensive ultrafiltration (Centriplus-YM3, Millipore, USA) by buffer exchange with $0.2 \%(w / v)$ SDS in PBS, pH 7.4. The amount of proteins was determined using the Bio-Rad Protein Assay kit (Bio-RAD, USA).

Polyclonal antibody production and dot blot analysis. Three female mice (BALB/C) were injected intraperitoneally with $100 \mu \mathrm{g}$ purified Pem-CHH1 dissolved in PBS, emulsified with an equal volume of Freund's adjuvant (Sigma, USA) (The total volume of the injection was $500 \mu \mathrm{l})$. The second and third immunizations with Pem-CHH1 in incomplete Freund's adjuvant were subsequently carried out every week after the first injection. On day five after the third injection, the sarcoma cell line (s180) was injected to induce the secretion of ascetic fluid. Either the ascetic fluid or the blood collected directly from the heart was incubated at $37^{\circ} \mathrm{C}$ for $1 \mathrm{~h}$. The antibody was collected by centrifugation at $3,000 \mathrm{~g}$ for $15 \mathrm{mins}$ and the supernatant was stored in aliquots at $-30^{\circ} \mathrm{C}$. The sensitivity and specificity of the antibody were determined by dot blot analysis.

Preparation of sinus gland extract. The neural tissues were dissected from eyestalks of $P$. monodon. The tissues were ground in liquid nitrogen using cold mortar and pestle. A phosphate buffer saline (PBS) (100 $\mu \mathrm{l} /$ one pair of eyestalk) was added to the ground tissues and homogenized thoroughly. The supernatant containing crude sinus gland extract was collected by centrifugation at $8,000 \mathrm{~g}$ for $20 \mathrm{~min}$ at $4^{\circ} \mathrm{C}$.

Biological assay with anti-Pem-CHH1 antibody-treated sinus gland extract. Farm-grown P. monodon $(\sim 20 \mathrm{~g})$ at intermolt stage were bilaterally eyestalk ablated and kept in the tank filled with artificial seawater (approximately $10 \mathrm{ppt}$ of salinity) for $18 \mathrm{~h}$ without feeding.

About fifty microliters of the haemolymph were collected from individual shrimp for measurement of baseline glucose level before injection was performed. $P$. monodon's sinus gland extract in PBS was pre-incubated with $1: 500$ dilution of anti-Pem-CHH 1 antibody from mouse no. 1 at $4^{\circ} \mathrm{C}$ for $2 \mathrm{~h}$. One pair equivalent of the treated sinus gland extract in a total volume of $100 \mu \mathrm{l}$ was injected into ten individual eyestalk-ablated $P$. monodon through the arthrodial membrane of the second walking leg. For the negative and positive control groups, each individual shrimp was injected with $100 \mu$ of sinus gland extract pre-incubated with $1: 500$ dilution of antibody to human growth hormone (anti-hGH antibody; received as a gift from Dr. Lily Eurwilaichitr) and untreated sinus gland extract, respectively. The fourth group of shrimp was injected with $100 \mu \mathrm{l}$ of PBS, the glucose level of which would represent the fluctuation due to stress from the injection. The haemolymph was collected from the shrimp at $30 \mathrm{~min}$ interval for $1.5 \mathrm{~h}$. after injection and frozen at $-30^{\circ} \mathrm{C}$. Before use, the haemolymph was centrifuged at $8,000 \times \mathrm{g}$ for $8 \mathrm{~min}$ and the glucose level in the haemolymph sample was determined by glucose dignostic kit (Sigma, USA). Statistical test of the data was performed by T-test (one population) (Microcal Origin, USA).

Biological assay with anti-Pem-CHH1 antibody. The experimental shrimp were cultured in aerated artificial sea water without eye stalk ablation. After 18 hour without feeding, $100 \mu \mathrm{l}$ of diluted antiPem-CHH 1 antibody was injected into twenty individual shrimp to a final dilution of 1:500 (approximate haemolymph volume of $\sim 20 \mathrm{~g}$ P. monodon is $1.5 \mathrm{ml}$ ) whereas the shrimp in the control group were injected with $100 \mu \mathrm{l}$ of PBS. The haemolymph was collected and determined for the glucose level as described above. The anti-Pem-CHH 1 antibody from mouse no. 1 was used in all biological assays.

\section{Results}

Expression and purification of recombinant Pem-CHH1. The Pem-CHH1 was expressed in E. coli as inclusion bodies that were mostly solubilized in $8 \mathrm{M}$ urea. The soluble fraction 


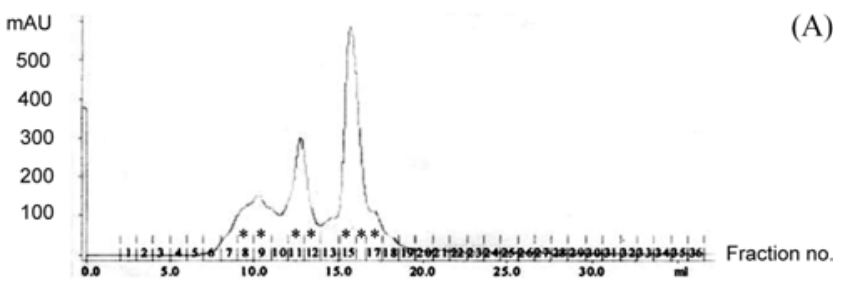

$\begin{array}{lllllllll}\mathrm{S} & \mathrm{M} & 1 & 2 & 3 & 4 & 5 & 6 & 7\end{array}$

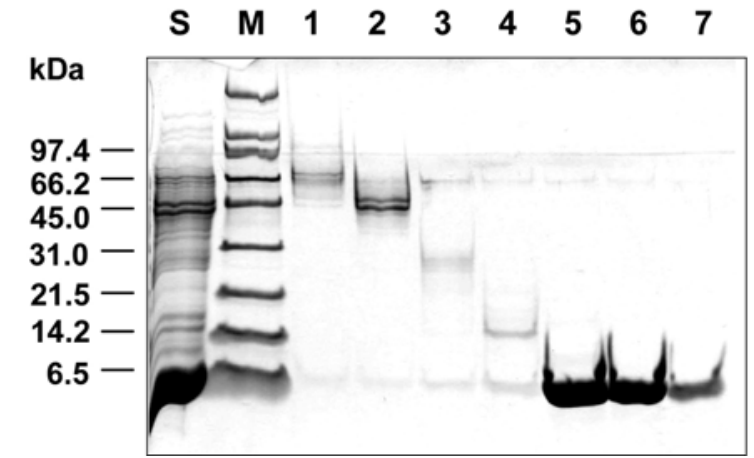

(B)

Fig. 2. Purification of recombinant Pem-CHH1. After solubilized in $8 \mathrm{M}$ urea $\mathrm{pH} 8.4$, the recombinant Pem-CHH1 was loaded into a Superdex-200 (HR-10/30, Global) gel filtration column. The proteins were eluted with $8 \mathrm{M}$ urea and fractions were collected at the flow rate of $0.25 \mathrm{ml} / \mathrm{min}$ (A) An elution profile of gel filtration. The proteins eluted from the column were detected by absorbance at $280 \mathrm{~nm}$. The asterisks mark the fractions to be analyzed by tricine SDS-PAGE. (B) Tricine SDSPAGE analysis of the gel filtration-purified fractions: fractions 8 , 9, 11, 12, 15, 16 and 17 (lanes 1-7, respectively). The soluble fraction of Pem-CHH1 before subjected to purification was loaded in lane $\mathrm{S}$. Lane $\mathrm{M}$ shows a broad-range protein marker (Bio-Rad).

of Pem-CHH1 was subsequently subjected to gel filtration FPLC chromatography, by which an approximate $8 \mathrm{kDa}$ protein band of purified Pem-CHH1 was obtained (Fig. 2). The final yield of the purified Pem-CHH 1 was $12 \mathrm{mg} / \mathrm{L}$. The purified Pem-CHH1 from fractions 15 to 17 (lanes 5-7, Fig. 2B) were pooled together and used as the antigen for immunization.

Sensitivity and specificity of anti-rPem-CHH1 antibody. The sensitivity of anti-Pem-CHH 1 antibodies from three mice was determined by dot blot analysis. Five nanograms of purified Pem-CHH 1 could be detected by a $1: 20,000$ dilution of the antibodies from all three mice whereas the lysate of 0.1 OD. E. coli cells did not give any detectable signal (data not shown). The anti-Pem-CHH1 antibody from mouse no. 1 was selected to be used in further experiments. This antibody could recognize the purified Pem-CHH1 whereas no cross-reactivity was detected with either structurally-related protein, Pem-MIH, or unrelated protein, human growth hormone (hGH), as shown by western blot analysis (Figure $3 \mathrm{~A})$. In addition to Pem-CHH1, the anti-Pem-CHH 1 antibody could recognize, albeit at a lesser extent, another two types of P. monodon's CHH, Pem-CHH2 and Pem-CHH3 (Fig. 3B).
(A)

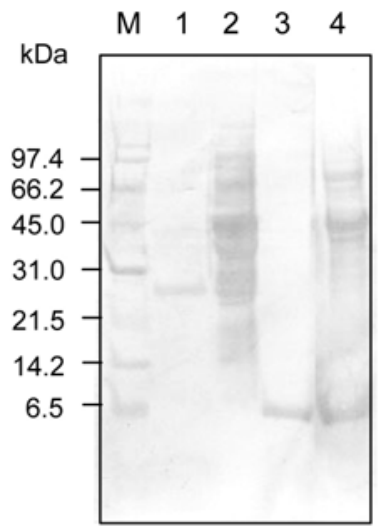

(B)
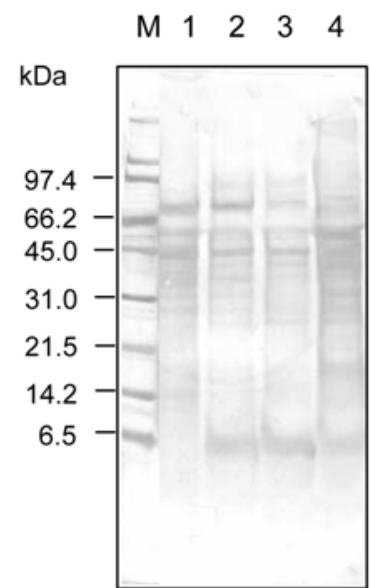

$\begin{array}{lllll}M & 1 & 2 & 3 & 4\end{array}$

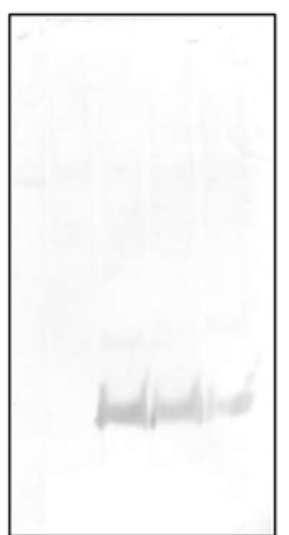

Fig. 3. Cross-reactivity of anti-Pem-CHH1 antibody. Specificity of anti-Pem-CHH1 antibody was tested by western blot analysis. The left panel in both (A) and (B) shows a Coomassie stainedpattern of the proteins on the membrane whereas the right panel shows the western blot detection by anti-Pem-CHH1 antibody of the corresponding membrane (A) Lane $\mathrm{M}$ shows a broad-range molecular weight marker. Lane 1 was loaded with $0.8 \mathrm{mg}$ human growth hormone (hGH); lane 2 with the lysate of 0.1 OD E. coli cells; lane 3 with $0.8 \mathrm{mg}$ purified Pem-CHH1. The crude recombinant Pem-MIH secreting from Pichia pastoris transformant was analyzed in lane 4. (B) Lane $M$ represents a broad-range molecular weight marker. Lane 1 shows the culture medium of $P$. pastoris containing pPICZaA expression vector only whereas the culture medium of $P$. pastoris containing Pem-CHH1, Pem$\mathrm{CHH} 2$ and Pem-CHH3 was loaded in lane 2, 3 and 4 , respectively.

Effect of anti-Pem-CHH1 antibody on hyperglycemic activity. After injection into eyestalk-ablated $P$. monodon, the extract from the sinus gland caused about two-fold increasing in the haemolymph glucose level within $0.5 \mathrm{~h}$. The haemolymph glucose remained steady at this high level until at least $1.5 \mathrm{~h}$. The hyperglycemia effect of the sinus gland extract was dramatically reduced when the extract was treated with antiPem-CHH1 antibody before injection. Comparing with the haemolymph glucose level at $0 \mathrm{hr}$, the hyperglycemia effect of the treated sinus gland extract was only about half of that induced by the untreated sinus gland extract. By contrast, the 


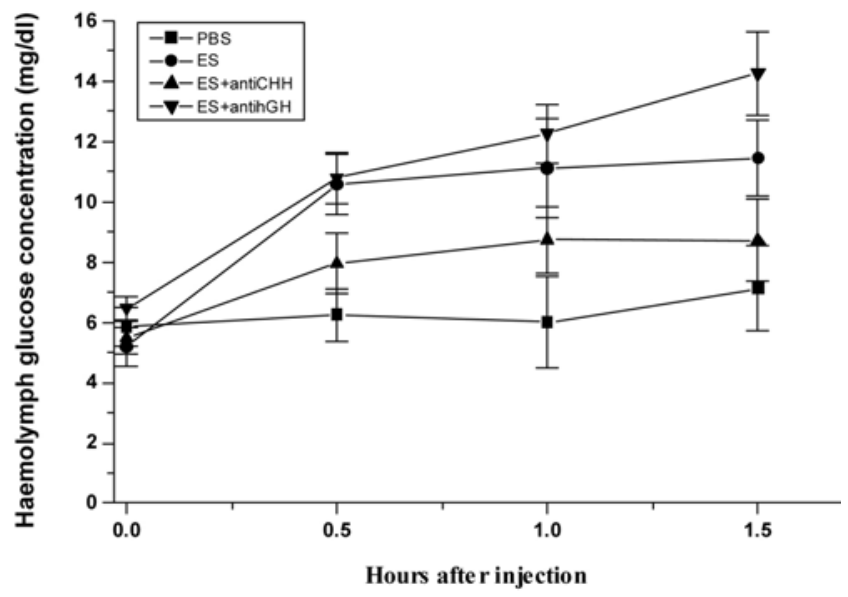

Fig. 4. The effect of anti-Pem-CHH1 antibody on hyperglycemia in $P$. monodon. Glucose levels in the haemolymph of $P$. monodon after injected with PBS (ם), eyestalk extract $(\mathbf{O})$, anti-Pem$\mathrm{CHH} 1$ treated eyestalk extract $(\boldsymbol{\Delta})$ and anti-hGH treated eyestalk extract $(\boldsymbol{\nabla})$ were shown. The error bars represent the SEM value $(\mathrm{n}=10)$.

shrimp injected with anti-hGH antibody treated sinus gland extract did not show any differences in the haemolymph glucose level from those injected with the sinus gland extract alone (Fig. 4).

In order to assay for the effect of anti-Pem-CHH1 antibody on $\mathrm{CHH}$ activity in_vivo, two groups of shrimp were injected with either PBS or the antibody. The result in Fig. 5 showed that following PBS injection, glucose level in the haemolymph remained constant over the period of $2 \mathrm{~h}$ compared with those before injection. However, injection of anti-Pem-CHH1 antibody showed a greater degree of reduction in the glucose level. Only about $70 \%$ of the basal level was detected at the first 30 min after injection, and a further reduction was observed until only half of the glucose level remained in the haemolymph at $2 \mathrm{~h}$ after injection (Fig. 5). Therefore, comparison between the glucose levels at each time point of the anti-Pem-CHH1 antibody-injected shrimp with that of PBS-injected shrimp revealed about $30-50 \%$ reduction in the percentage of glucose concentration.

\section{Discussion}

To date, at least eight crustacean hyperglycemic hormones have been reported in P. monodon. These can be divided into two subgroups: Pem-CHH1 to 3 (Treerattrakool et al., 2002; Udomkit et al., 2004) and Pm-sgp-I to V (Davey et al., 2000). In this study, Pem-CHH1 was expressed in E. coli and used for antibody production in mice. This anti-Pem-CHH1 antibody could recognize not only Pem-CHH1 but also Pem$\mathrm{CHH} 2$ and 3. This is not surprising since the mature peptides of the three types of Pem-CHH share more than $90 \%$ homology in their amino acid sequences with most of the

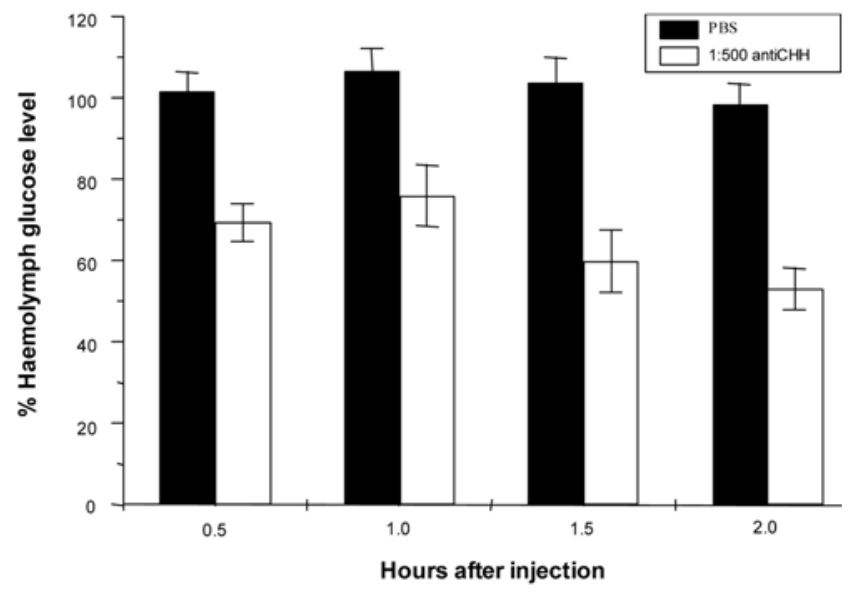

Fig. 5. A time course of changes in the glucose level after injection with PBS (solid bar) and anti-Pem-CHH1 antibody (open bar). The values were represented as percentages of glucose in the haemolymph compared with the basal level at $0 \mathrm{~h}$ $(100 \%)$ in each group of shrimp.

differences lying within the first five N-terminal amino acid residues.

Sithigorngul et al., 2002 has demonstrated that the antibody raised against 15 amino acid residues at the C-terminus of Pem-CHH1 (previously called Pem-CMG) did not cross-react with the antibody made against $\mathrm{C}$-terminus of $\mathrm{CHH}$ of Macrobrachium rosenbergii, which recognized proteins fractions from $P$. monodon's eyestalk that contain hyperglycemic activity. Interestingly, the C-termini of $\mathrm{CHH}$ from $M$. rosenbergii and Pm-sgp-I to V of P. monodon_are more similar to each other than to that of Pem-CHH1 to 3 , therefore the antibodies were presumed to be able to distinguish the peptides at their C-terminal sequences. This assumption can also explain the failure of anti-Pem-CHH1 to detect its structurally related peptide, Pem-MIH. Although $\mathrm{CHH}$ and $\mathrm{MIH}$ are similar in their overall structure, the amino acid sequences of Pem-CHH and Pem-MIH (Yodmuang et al., 2004) are also different at the C-termini. Moreover, the solution structure of MIH of Marsopenaeus japonicus elucidated by NMR showed distinctive characteristics from that of the homology-modeled structure of $\mathrm{CHH}$ of the same species (Katayama et al., 2003). The $\mathrm{MIH}$ contains an additional a-helix at the $\mathrm{N}$ terminus and the protruding $\mathrm{C}$ terminal tail, which are sterically closed to each other whereas $\mathrm{CHH}$ lacks these features. This site may play significant role in conferring activity of the hormones and thus be responsible for the specificity of the antibody produced against Pem-CHH1.

When the eyestalk extract was treated with anti-PemCHH1 antibody before injected into the shrimp, it lost about half of its hyperglycemic activity compared to that of the untreated eyestalk extract (Fig. 4). This result suggested that the impaired hyperglycemia was due to the removal of $\mathrm{CHH}$ fraction in the eyestalk extract by the antibody. Similar experiments have been performed in the shrimp, Palaemon 
elegans, in which the antibody to the $\mathrm{CHH}$ of the Norway lobster, Nephrops norvegicus, could deplete the hyperglycemic response to the level comparable to that when injected with extraction buffer alone (Giulianini et al., 2002). However, in their experiments the eyestalk extract was incubated with $1: 20$ dilution of antibody whereas in our study the extract was mixed with $1: 500$ dilution of the antibody. The effect of the antibody on hyperglycemic activity of the hormone may be dose-dependent, and higher level of inhibition is expected if higher concentration of antibody was used. Moreover, the remaining activity could come from other isoforms of $\mathrm{CHH}$ in $P$. monodon that were not recognized by anti-Pem-CHH1 antibody, presumably Pm-sgp I to $\mathrm{V}$, although there is no direct evidence for the hyperglycemic activity of these hormones so far.

Direct injection of anti-Pem-CHH1 antibody into P. monodon could reduce hyperglycemic activity in the haemolymph by $30-50 \%$, which was significantly different from the basal activity in PBS-injected shrimp. This inhibitory effect demonstrated that the antibody could recognize the hormone and inhibit the hormone action in vivo.

Finally, since $\mathrm{CHH}$ activity triggers important events in some physiological processes, especially molting and ovarian maturation (Yasuda et al., 1994; Gu et al., 2000), the in vivo inhibition of this hormone by specific antibody demonstrated here therefore provide a promising strategy to modulate growth and reproduction of economically important species such as $P$. monodon.

Acknowledgments We thank Dr. Witoon Tirasophon and Mrs. Somsri Sakdee for their help in antibody preparation. This work was financially supported by the Thailand Research Fund (TRF).

\section{References}

Avarre, J. C., Khayat, M., Michelis, R., Nagasawa, H., Tietz, A. and Lubzens, E. (2001) Inhibition of de novo synthesis of a jelly layer precursor protein by crustacean hyperglycemic hormone family peptides and posttranscriptional regulation by sinus gland extracts in Penaeus semisulcatus ovaries. Gen. Comp. Endocrinol. 124, 257-268.

Chang, E. S. and O'Connor, J. D. (1985) Metabolism and transport of carbohydrates and lipids; in The Biology of Crustacea, Mantel, L. H. (ed.), pp. 263-281, Academic Press, New York, USA.

Charmantier, G., Charmantier-Daures, M. and Van Herp, F. (1997) Hormonal regulation of growth and reproduction in crustaceans; in Endocrinology and Reproduction, Recent Advances in Marine Biotechnology, Fingerman, M., Nagabhushanam, R. and Thompson, M-F. (eds.), pp. 109-161, Science Publishers Inc., New Hampshire, USA.

Charmantier-Daures, M., Charmantier, G., Jansen, K. P. C., Aiken, D. E. and Van Herp, F. (1994) Involement of eyestalk factors in the neuroendocrine control of osmoregulation in adult American lobster, Homarus americanus. Gen. Comp.
Endocrinol. 94, 281-293.

Cooke, J. M. and Sullivan, R. E. (1982) Hormones and neurosecretion; in The Biology of Crustacea, Bliss, D. E. (ed.), pp. 205-290, Academic Press, New York, USA

Davey, M. L., Hall, M. R., Willis, R. H., Oliver, R. W., Thurn, M. J. and Wilson, K. J. (2000) Five crustacean hyperglycemic family hormones of Penaeus monodon: complementary DNA sequence and identification in single sinus glands by electrospray ionization-fourier transform mass spectroscopy. Mar. Biotechnol. 2, 80-91.

Endo, H., Nagasawa, H. and Watanabe, T. (2000) Isolation of a cDNA encoding a CHH-family peptide from the silkworm Bombyx mori. Insect. Biochem. Mol. Biol. 30, 355-361.

Giulianini, P. G., Pandolfelli, N., Lorenzon, S., Ferrero, E. and Edomi, P. (2002) An antibody to recombinant crustacean hyperglycemic hormone of Nephrops norvegicus cross-reacts with neuroendocrine organs of several taxa of malacostracan Crustacea. Cell Tissue Res. 307, 243-254.

Gu, P-L., Yu, K. L. and Chan, S-M. (2000) Molecular characterization of an additional shrimp hyperglycemic hormone: cDNA cloning, gene organization, expression and biological assay of recombinant proteins. FEBS Lett. 472, 122128.

Katayama, H., Nagata, K., Ohira, T., Yumoto, F. and Tanokura, M. (2003) The solution structure of molt-inhibiting hormone from the Kuruma prawn Marsupenaeus japonicus. J. Biol. Chem. 278, 9620-9623.

Keller, R. (1992) Crustacean neuropeptides: structures, functions and comparative aspects. Experientia. 48, 439-448.

Khayat, M., Yang, W-J., Aida, K., Nakasawa, H., Tiezt, A., Funkenstein, B. and Lubzens, E. (1998) Hyperglycemic hormones inhibit protein and mRNA synthesis in in vitro incubated ovarian fragments of the marine shrimp Penaeus semisulcatus. Gen. Comp. Endrocrinol. 110, 307-318.

Meredith, J., Cheng, N., Little Ford, D., Ring, M., Audsley, N., Brock, H. and Phillips J. E. (1995) Amino acid sequence of locust ion transport peptide (ITP). FASEB J. 9, 355.

Santos, E. A., Nery, L. E. M., Keller, R. and Goncalves, A. A. (1997) Evidence for the involvement of the crustacean hyperglycemic hormone in the regulation of lipid metabolism. Physiol. Zool. 70, 415-420.

Sedlmeier, D. (1982) The mode of acion of the crustacean neurosecretory hyperglycemic hormone (CHH). II. Involvement of glycogen synthase. Gen. Comp. Endocrinol. 47, 426-432.

Serrano, L., Blanvillain, G., Soyez, D., Charmantier, G., Grousset, E., Aujoulat, F. and Spanings-Pierrot, C. (2003) Putative involvement of crustacean hyperglycemic hormone isoforms in the neuroendocrine mediation of osmoregulation in the crayfish Astacus leptodactylus. J. Exp. Biol. 206, 979-988.

Sithigorngul, P., Panchan, N., Chaivisuthangkura, P., Longyant, S., Sithigorngul, W. and Petsom, A. (2002) Differential expression of $\mathrm{CMG}$ peptide and crustacean hyperglycemic hormones (CHHs) in the eyestalk of the giant tiger prawn Penaeus monodon. Peptides 23, 1943-1952.

Treerattrakool, S., Eurwilaichitr, L., Udomkit, A. and Panyim, S. (2002) Secretion of Pem-CMG, a peptide in the CHH/MIH/ GIH family of Penaeus monodon, in Pichia pastoris is directed by secretion signal of the a-mating factor from Saccharomyces cerevisiae. J. Biochem. Mol. Biol. 35, 476-481.

Treerattrakool, S., Udomkit, A., Eurwilaichitr, L., Sonthayanon, B. 
and Panyim S. (2003) Expression of biologically active crustacean hyperglycemic hormone (CHH) of Penaeus monodon in Pichia pastoris. Mar. Biotechnol. 5, 373-379.

Udomkit, A., Treerattrakool, S. and Panyim, S. (2004) Crustacean hyperglycemic hormone of Penaeus monodon: cloning, production of active recombinant hormones and their expression in various shrimp tissues. J. Exp. Mar. Biol. Ecol. 298, 79-91.

Yasuda, A., Yasuda, Y., Fujita, T. and Naya, Y (1994)
Characterization of crustacean hyperglycemic hormone from the crayfish (Procambarus clarkii): multiplicity of molecular forms by sterioinversion and diverse function. Gen. Comp. Endocrinol. 95, 387-398.

Yodmuang S., Udomkit A., Treerattrakool S. and Panyim S. (2004) Molecular and biological characterization of moltinhibiting hormone of Penaeus monodon. J. Exp. Mar. Biol. Ecol. 312, 101-114. 